\title{
Development of a minimally invasive pulmonary porcine embolism model via endobronchial ultrasound
}

\author{
Terunaga Inage ${ }^{1,2}$, Kosuke Fujino ${ }^{1}$, Yamato Motooka ${ }^{1}$, Tsukasa Ishiwata ${ }^{1}$, Hideki Ujiie ${ }^{1}$, \\ Alexander Gregor ${ }^{1}$, Nicholas Bernards ${ }^{1}$, Harley H. L. Chan ${ }^{3}$, Zhenchian Chen ${ }^{1}$, Masato Aragaki ${ }^{1}$, \\ Tomonari Kinoshita ${ }^{1}$, Andrew Effat ${ }^{1}$, Ichiro Yoshino ${ }^{2}$, Kazuhiro Yasufuku ${ }^{1}$ \\ ${ }^{1}$ Division of Thoracic Surgery, Toronto General Hospital, University Health Network, Toronto, Canada; ${ }^{2}$ Department of General Thoracic Surgery, Chiba \\ University Graduate School of Medicine, Chiba, Japan; ${ }^{3}$ TECHNA Institute, University Health Network, Toronto, Canada \\ Contributions: (I) Conception and design: T Inage, K Fujino, K Yasufuku; (II) Administrative support: T Inage, N Bernards, K Yasufuku; (III) Provision of \\ study materials or patients: T Inage, A Gregor, K Fujino, Y Motooka, T Ishiwata, N Bernards, K Yasufuku; (IV) Collection and assembly of data: T Inage, K \\ Fujino, Y Motooka, T Ishiwata, H Ujiie, A Gregor, N Bernards, HHL Chan, Z Chen, M Aragaki, T Kinoshita, A Effat; (V) Data analysis and interpretation: \\ T Inage, K Fujino, A Gregor, I Yoshino, K Yasufuku; (VI) Manuscript writing: All authors; (VII) Final approval of manuscript: All authors. \\ Correspondence to: Kazuhiro Yasufuku, MD, PhD. Head, Division of Thoracic Surgery, University Health Network; FG Pearson-RJ Ginsberg Chair \\ in Thoracic Surgery, William Coco Chair in Surgical Innovation for Lung Cancer, Senior Scientist, Toronto General Hospital Research Institute; \\ Director of Endoscopy, University Health Network; Director, Interventional Thoracic Surgery Program, University Health Network; Professor and \\ Chair, Division of Thoracic Surgery, University of Toronto, Toronto M5G2C4, Canada. Email: kazuhiro.yasufuku@uhn.ca.
}

Background: Current massive pulmonary embolism (PE) animal models use central venous access to deliver blood clots, which have features of random clot distribution and potentially fatal hemodynamic compromise. A clinically relevant preclinical model for generating pulmonary emboli in a more controlled fashion would be of value for a variety of research studies, including initial evaluation of novel therapeutic approaches. Endobronchial ultrasound-guided transbronchial needle injection (EBUS-TBNI) is a newly established approach for peri-tracheal/bronchial targets. The purpose of the present work was to establish a minimally invasive PE model in swine via a transbronchial approach.

Methods: In anesthetized Yorkshire pigs, a 21-G EBUS-guided transbronchial needle aspiration (EBUSTBNA) needle was introduced into the pulmonary artery under EBUS guidance. Autologous blood clots were administered into the right and left lower pulmonary arteries sequentially (PE1 and PE2, respectively). Hemodynamic and biochemical responses were evaluated.

Results: Ten pigs were evaluated; all 20 blood clots $(6.3 \pm 1.9 \mathrm{~mL})$ were successfully injected. After injection, mean pulmonary artery pressure (mPAP; $\mathrm{mmHg}$ ) increased (baseline: $16.6 \pm 5.6$ vs. $\mathrm{PE} 1: 24.5 \pm 7.6, \mathrm{P}<0.0001$ vs. $\mathrm{PE} 2: 26.9 \pm 6.7, \mathrm{P}<0.0001)$, and a positive correlation was observed between clot volume and change in mPAP (PE1: $r=0.69, \mathrm{P}=0.025$; PE1 + PE2: $\mathrm{r}=0.60, \mathrm{P}=0.063)$. Mean arterial pressure (MAP; mmHg) (baseline: 57.5 \pm 5.1 vs. $\mathrm{PE} 1: 59.0 \pm 9.1, \mathrm{P}=0.918$ vs. $\mathrm{PE2}$ : $60.9 \pm 9.6, \mathrm{P}=0.664)$ remained stable. No complications were observed.

Conclusions: EBUS allows minimally invasive, precise, and reliable generation of pulmonary emboli in pigs. This model may serve as an important tool for new PE-related diagnostic and therapeutic research.

Keywords: Pulmonary embolism (PE); swine; endobronchial ultrasound (EBUS)

Submitted Jul 29, 2021. Accepted for publication Dec 28, 2021.

doi: $10.21037 /$ jtd-21-1242

View this article at: https://dx.doi.org/10.21037/jtd-21-1242

(c) Journal of Thoracic Disease. All rights reserved. 


\section{Introduction}

Establishment of clinically relevant pulmonary embolism (PE) animal models is crucial for translational research. Current PE animal models use central venous access to pharmacologically stimulate thrombus formation (1) or deliver autologous blood clots (2). These models replicate the disease state well with features of random clot distribution $(1,2)$ and potentially fatal hemodynamic compromise $(3,4)$. However, this variability can make these models challenging. Endobronchial ultrasoundguided transbronchial needle injection (EBUS-TBNI) is an approach for treatment of peri-bronchial lesions (5-7). Transbronchial injection provides potential advantages over prior methods and enables the ability to control the embolus site, embolus size, and number of emboli. Controlled generation of pulmonary emboli would be of value for a range of research, including initial evaluation of novel therapeutic approaches. The aim of the study was to establish a minimally invasive PE model in swine via EBUSTBNI. We present the following article in accordance with the ARRIVE reporting checklist (available at https://jtd. amegroups.com/article/view/10.21037/jtd-21-1242/rc).

\section{Methods}

\section{Instrumentation and measurements}

Experiments were performed under a project license (No. AUP5928) granted by the University Health Network Animal Care Committee, in compliance with the institutional guidelines for the care and use of animals. A protocol was prepared before the study without registration. Yorkshire pigs (mean $33 \mathrm{~kg}$; Table S1) were used in this study. Following preanesthetic sedation with intramuscular ketamine (20 mg/kg, Bimeda, Canada), midazolam (0.3 mg/kg, Fresenius Kabi, Toronto, Canada), and atropine (0.04 mg/kg, CDMV Inc., Québec, Canada) general anesthesia was achieved with isoflurane (Fresenius Kabi). Animals were placed supine, intubated and ventilated as follows: fraction of inspired oxygen $50 \%$, tidal volume $7 \mathrm{~mL} / \mathrm{kg}$, respiratory rate 14 breath/min, and zero end-expiratory pressure. Oxygen saturation $\left(\mathrm{SpO}_{2}\right)$, heart rate $(\mathrm{HR})$ and end-tidal $\mathrm{CO}_{2}\left(\mathrm{ETCO}_{2}\right)$ were monitored throughout. Tidal volume and respiratory rate were adjusted to achieve baseline $\mathrm{ETCO}_{2}$ 35-45 $\mathrm{mmHg}$ and $\mathrm{SpO}_{2}$ 94-100\%. A 9-French (F) sheath (I505BF9; Edwards Lifesciences Corp., Irvine, CA, USA) was introduced into the right femoral vein. This sheath was used for both blood collection and introduction of a $7 \mathrm{~F}$ Swan-Ganz catheter (111F7; Edwards Lifesciences Corp.), which was itself advanced into the main pulmonary artery under fluoroscopic guidance. Lateral to the sheath, a 20-G angiocath was placed in the femoral artery. Mean arterial pressure (MAP) and mean pulmonary artery pressure (mPAP) were monitored and recorded. Arterial blood gases and lactate were measured using a blood gas analyzer (RAPIDPoint 500; Siemens Healthcare, Erlangen, Germany).

\section{Preparation of pulmonary emboli}

Emboli were formed ex vivo from autologous blood collected in $30 \mathrm{~mL}$ syringes (BD 309650; Becton, Dickinson and Company, Franklin Lakes, NJ, USA) (Figure 1A). Blood-filled syringes were incubated for $2 \mathrm{~h}$ at $37{ }^{\circ} \mathrm{C}$ without agitation or pharmaceutical accelerant (LR19654; Foster Refrigerator, New York, NY, USA) (Figure 1B). After incubation, clot was transferred into $1 \mathrm{~mL}$ syringes (BD 309628; Becton, Dickinson and Company) for injection (Figure 1C,1D).

\section{Experimental protocol}

All animals underwent the protocol shown in Figure 2. Baseline (B) values were recorded $170 \mathrm{~min}$ after anesthetic induction. Approximately $10 \mathrm{~min}$ after clot injection 1 (PE1) and 10 min after clot injection 2 (PE2), additional measurements were documented. PE1 and PE2 were administered sequentially. Initially, $3 \mathrm{~mL}$ of clot was injected randomly into the right or left lower lobe pulmonary artery via EBUS-TBNI (see below). Successful intravascular injection of clot was confirmed by EBUS, with Doppler as needed. If no embolus was clearly seen, additional $1 \mathrm{~mL}$ clots were administered until visualized. The procedure was repeated in the contralateral lung $20 \mathrm{~min}$ after PE1. The total observation time for all animals was $2 \mathrm{~h}$ after PE2, including airway evaluation by bronchoscopy (BF-UC180F or BF-160; Olympus, Tokyo, Japan). Pigs were then either sacrificed or used for further study, when possible.

\section{EBUS and ultrasound processor}

Convex-probe EBUS (BF-UC180F; Olympus) was performed with a dedicated ultrasound processor (EU-ME1; Olympus). Emboli were injected using a 21-G EBUSguided transbronchial needle aspiration (EBUS-TBNA) 

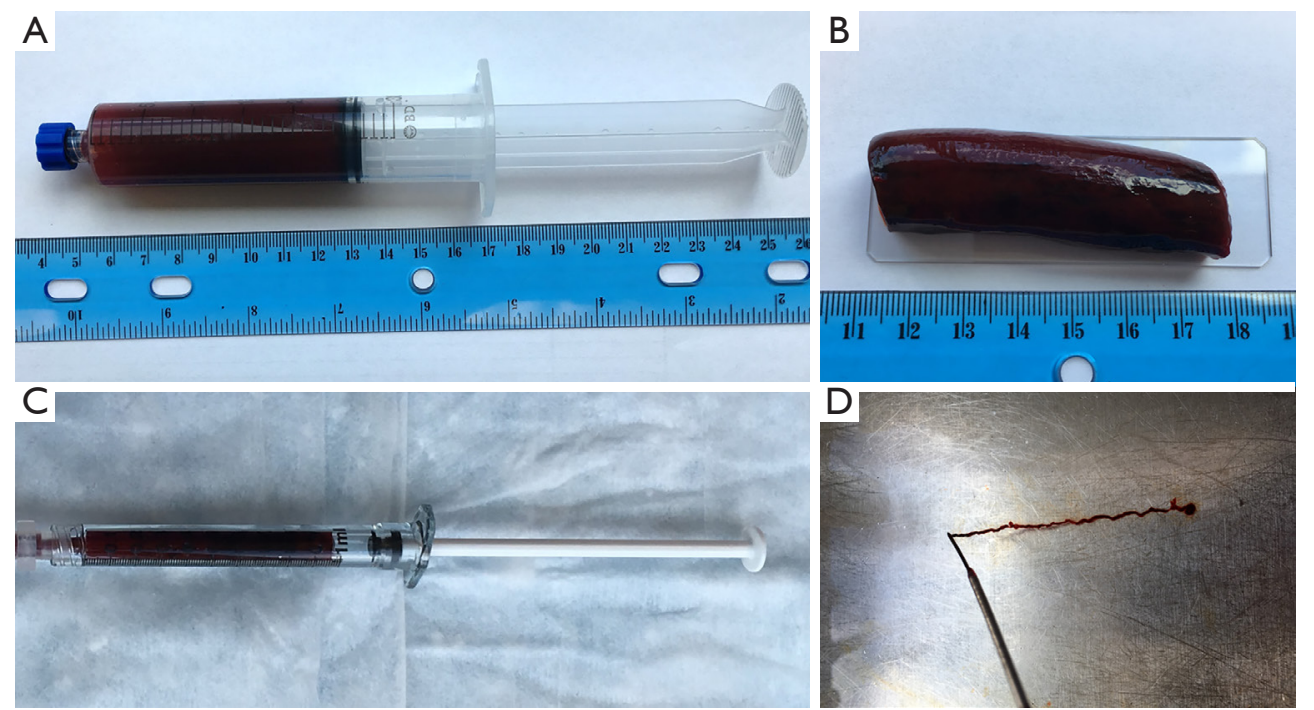

Figure 1 Ex vivo autologous blood clot formation. Blood (A) is incubated $2 \mathrm{~h}$ at $37^{\circ} \mathrm{C}$ in a syringe to encourage the formation of solid clot (B). Clot 'aliquots' are then transferred into $1 \mathrm{~mL}$ syringes $(\mathrm{C})$ for administration via a 21-G EBUS-TBNA needle (D). EBUS-TBNA, endobronchial ultrasound-guided transbronchial needle aspiration.

\begin{tabular}{|c|c|c|c|c|c|c|}
\hline Preparation & Baseline & Embolus 1 (PE1) & Evaluation 1 & Embolus 2 (PE2) & Evaluation 2 & End \\
\hline $150 \mathrm{~min}$ & $170 \mathrm{~min}$ & $180 \mathrm{~min}$ & $190 \mathrm{~min}$ & $200 \mathrm{~min}$ & $210 \mathrm{~min}$ & $330 \mathrm{~min}$ \\
\hline $\begin{array}{l}\text { Anesthetic induction } \\
\text { Intubation } \\
\text { Vascular line placement } \\
\text { Collect blood sample } \\
\text { Clot formation ( } 2 \mathrm{~h})\end{array}$ & $\begin{array}{l}\text { Record baseline } \\
\text { hemodynamics } \\
\text { parameters } \\
\text { Collect baseline } \\
\text { arterial sample }\end{array}$ & $\begin{array}{c}\text { Administer emboli } \\
\# 1\end{array}$ & $\begin{array}{c}\text { Record post-PE1 } \\
\text { hemodynamics } \\
\text { parameters } \\
\text { Collect post-PE1 } \\
\text { arterial sample }\end{array}$ & $\begin{array}{c}\text { Administer emboli } \\
\# 2\end{array}$ & $\begin{array}{c}\text { Record post-PE2 } \\
\text { hemodynamics } \\
\text { parameters } \\
\text { Collect post-PE2 } \\
\text { arterial sample }\end{array}$ & $\begin{array}{c}\text { Monitor for } \\
\text { complications }\end{array}$ \\
\hline
\end{tabular}

Figure 2 Study protocol.

needle (NA-201SX-4021; Olympus).

\section{Administration of pulmonary emboli}

The pulmonary artery was visualized by EBUS (Figure $3 A$ ), including Doppler (Figure 3B). An optimal injection site was selected; features of an ideal injection site included visualization of pulmonary artery branching and a clear path to the artery. Lower lobe arteries were used in this study due to ease of access and clearer visualization. A 21-G EBUS-TBNA needle was advanced into the pulmonary artery branch under ultrasound guidance (Figure 3C). Confirmation of successful arterial puncture was verified by blood backflow with aspiration of the TBNA needle. Once backflow was confirmed, a pre-formed autologous blood clot was administered into the pulmonary artery using realtime EBUS guidance (Figure 3D).

\section{Ultrasonic and radiological imaging and pathologic evaluation of pulmonary emboli}

Post-PE EBUS confirmed the presence of pulmonary emboli at the planned injection sites (B-mode; Figure 3E) with disruption of blood flow (Doppler; Figure $3 F$ ). Pulmonary angiography (Figure $4 A$ ) and contrast-enhanced cone beam computed tomography (Figure $4 B$ ) performed in representative pigs demonstrated intravascular filling defects consistent with emboli. Post-mortem examination 

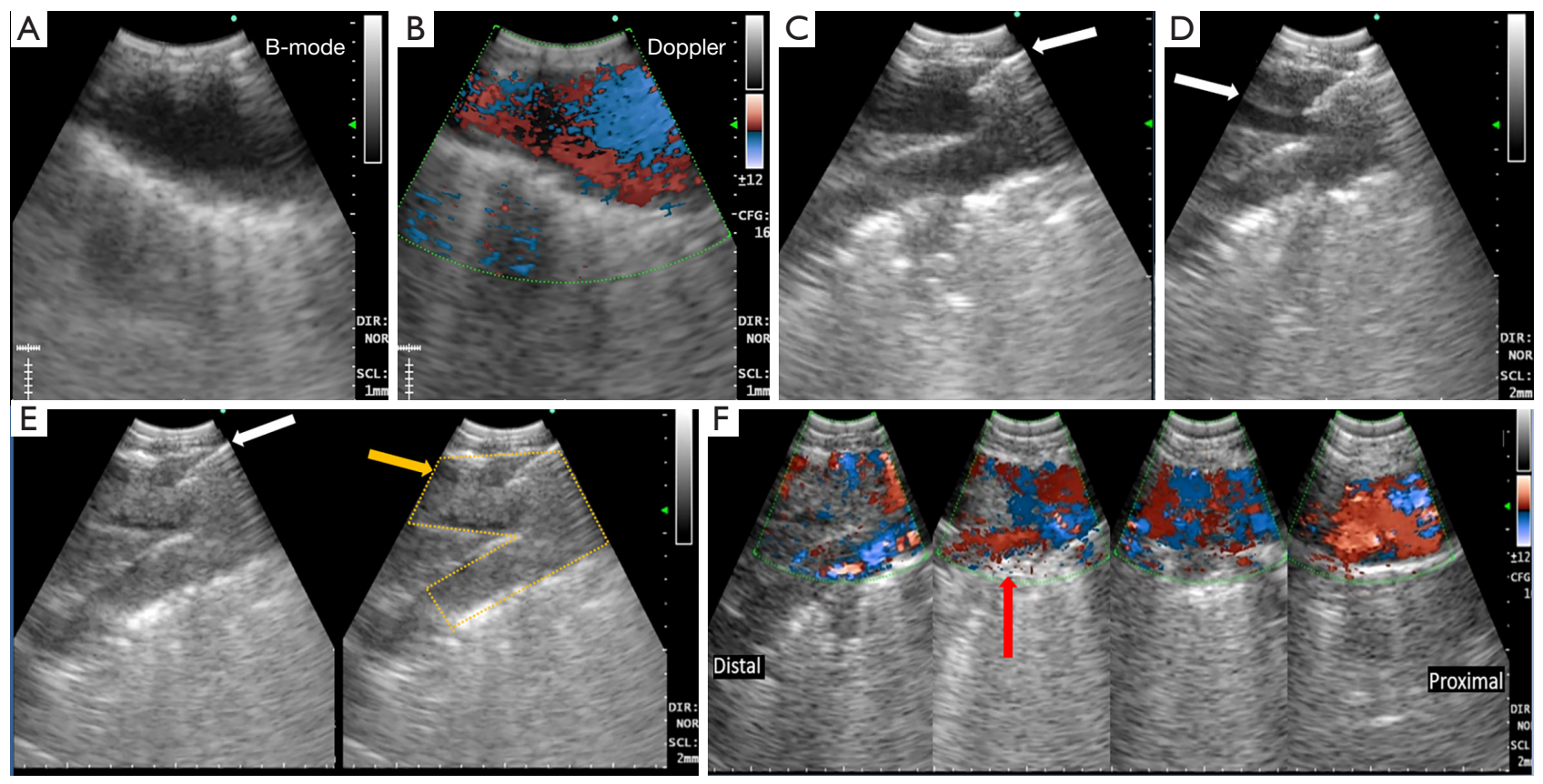

Figure 3 EBUS-TBNI for generation of pulmonary emboli. (A) EBUS B-mode and (B) Doppler images identify the pulmonary artery. (C) Puncture of the artery by the 21-G EBUS-TBNA needle is monitored using B-mode. (D) Intravascular clot injection is monitored in realtime using EBUS; expelled clot is noted by the white arrow. (E) After injection, a large clot is seen on EBUS B-mode (yellow box and arrow). (F) Disrupted arterial flow is confirmed by EBUS Doppler (red arrow marks proximal edge of embolus). EBUS-TBNI, endobronchial ultrasound-guided transbronchial needle injection; EBUS-TBNA, endobronchial ultrasound-guided transbronchial needle aspiration.

revealed significant pulmonary emboli (Figure $4 C$ : the emboli was observed after injection of $7 \mathrm{~mL}$ clot into the right lower lobe pulmonary artery). Microscopic examination demonstrated large arterial emboli with adjacent hemorrhagic regions consistent with pulmonary infarction (Figure 4D)

\section{Statistical analysis}

A repeated measures ANOVA with Tukey's Honest Significant Difference test was used to compare baseline and post-clot injection data. Relation between injected clot volume and mPAP was analyzed by linear regression and correlation. A P value of $<0.05$ was considered statistically significant. Statistical analysis was performed using GraphPad Prism 8.0 (GraphPad Software ver.9, San Diego, CA, USA, RRID:SCR_002798). Values are expressed below as mean \pm standard error of the mean (SEM).

\section{Pathologic evaluation}

Lungs and emboli were collected from euthanized animals and fixed in $10 \%$ formalin. Representative slides from embolized and normal lung were stained with hematoxylin and eosin.

\section{Results}

In 10 pigs, all 20 blood clots (average clot size $6.6 \pm 2.3 \mathrm{~mL}$, Table S1) were successfully injected. Hemodynamic and biochemical parameters are summarized in Table 1. Baseline mPAP was $16.6 \pm 5.6 \mathrm{mmHg}$. After clot administration, mPAP increased to $24.5 \pm 9.1$ ( $\mathrm{B} v s$. $\mathrm{PE} 1, \mathrm{P}<0.0001$ ) and $26.9 \pm 6.7 \mathrm{mmHg}(\mathrm{B} v s . \mathrm{PE} 2, \mathrm{P}<0.0001)$ (Figure 5). Lactate $(\mathrm{mmol} / \mathrm{L})(\mathrm{B}: 0.60 \pm 0.2$ vs. $\mathrm{PE} 2: 0.86 \pm 0.3, \mathrm{P}=0.007)$ also changed significantly after PE2. Other parameters changed slightly after injection, including HR (beats/min) (B: $93.3 \pm 12.3$ vs. $\mathrm{PE} 2: 113 \pm 30, \mathrm{P}=0.061$ ), partial pressure of arterial carbon dioxide $\left(\mathrm{PaCO}_{2} ; \mathrm{mmHg}\right)(\mathrm{B}: 47.2 \pm 12.5$ vs. PE2: $53.9 \pm 8.8, \mathrm{P}=0.062)$, and partial pressure of arterial oxygen $\left(\mathrm{PaO}_{2} ; \mathrm{mmHg}\right)(\mathrm{B}: 155.5 \pm 49.6$ vs. $\mathrm{PE} 1: 127.7 \pm 27.5$, $\mathrm{P}=0.094$ vs. $\mathrm{PE} 2: 125.1 \pm 37.2, \mathrm{P}=0.087)$, but there were no significant statistical differences observed. Serial monitoring out to 120 min showed stability in all hemodynamic 


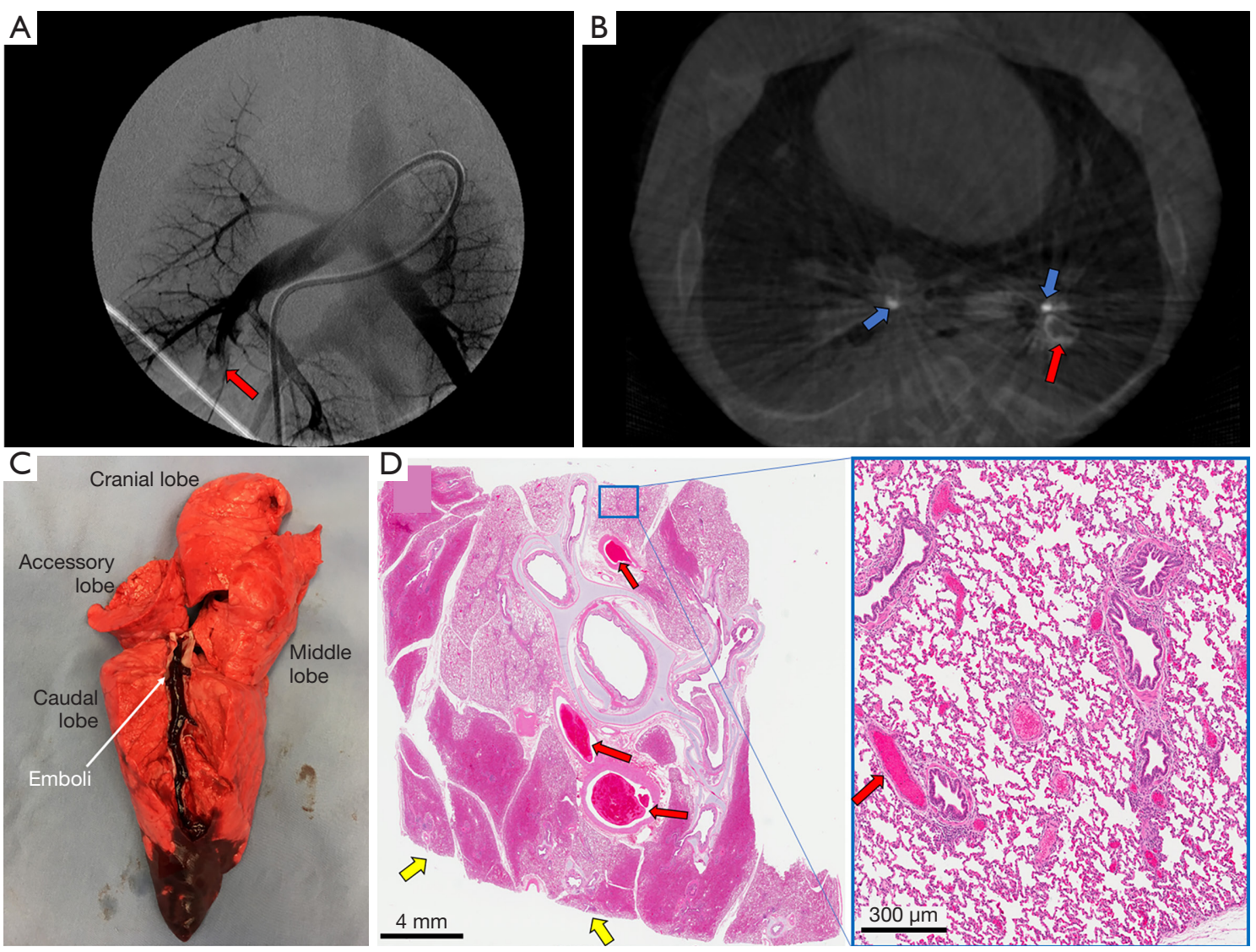

Figure 4 Radiological and pathological evaluation of pulmonary emboli. (A) Pulmonary artery angiography demonstrates a filling defect (red arrow), confirming clot formation. (B) Contrast-enhanced cone beam computed tomography similarly demonstrates a filling defect (red arrow; pulmonary artery catheter indicated by blue arrow). (C) Embolization of the pulmonary artery was confirmed by postmortem examination; this specific embolus followed injection of $7 \mathrm{~mL}$ clot into the right lower lobe pulmonary artery. (D) Pathological evaluation reveals large arterial emboli (red arrows) with adjacent regions of hemorrhagic pulmonary infarction (yellow arrows); staining method: hematoxylin and eosin staining; scale bar corresponds to $4 \mathrm{~mm}$ (left) and $300 \mu \mathrm{m}$ (right).

Table 1 Summary of hemodynamic and biochemical measurements

\begin{tabular}{|c|c|c|c|c|c|}
\hline Parameters & Baseline & PE1 & PE2 & \multicolumn{2}{|c|}{$P$ value } \\
\hline HR (beat/min) & $93.3 \pm 12.3$ & $95.2 \pm 10.9$ & $113 \pm 30$ & 0.875 & 0.061 \\
\hline MAP $(\mathrm{mmHg})$ & $57.5 \pm 5.1$ & $59.0 \pm 9.1$ & $60.9 \pm 9.6$ & 0.918 & 0.664 \\
\hline mPAP $(\mathrm{mmHg})$ & $16.6 \pm 5.6$ & $24.5 \pm 7.6$ & $26.9 \pm 6.7$ & $<0.0001$ & $<0.0001$ \\
\hline $\mathrm{PaO}_{2}(\mathrm{mmHg})$ & $155.5 \pm 49.6$ & $127.7 \pm 27.5$ & $125.1 \pm 37.2$ & 0.094 & 0.087 \\
\hline Lactate (mmol) & $0.60 \pm 0.2$ & $0.76 \pm 0.3$ & $0.86 \pm 0.3$ & 0.174 & 0.007 \\
\hline
\end{tabular}

Data are presented as mean \pm SEM. Baseline and post-clot injection (PE1 or PE2) parameters were compared using a repeated measures ANOVA followed by Tukey's Honest Significant Difference test. PE1 and PE2, 10 min after PE1 and PE2 administration. HR, heart rate; MAP, mean arterial pressure; mPAP, mean pulmonary artery pressure; $\mathrm{PaCO}_{2}$, partial pressure of carbon dioxide; $\mathrm{PaO}_{2}$, partial pressure of oxygen; SEM, standard error of the mean. 

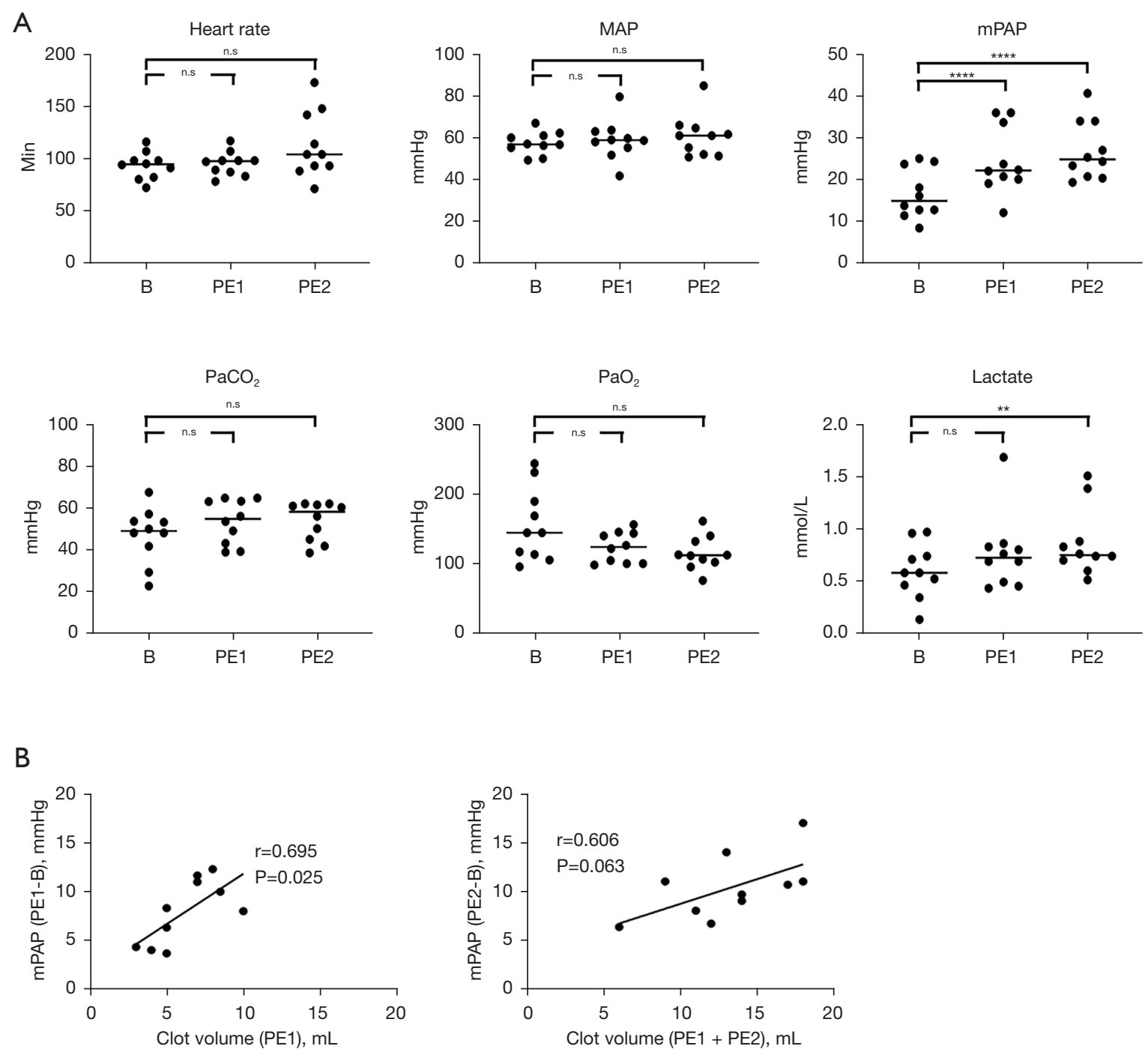

Figure 5 Changes in hemodynamics and biochemistry post-clot injection. (A) HR, MAP, mPAP, $\mathrm{PaCO}_{2}, \mathrm{PaO}_{2}$, and lactate are shown from $\mathrm{B}, \mathrm{PE} 1$ and PE2. Data presented as mean \pm SEM. (B) Injected clot volume (PE1 or PE1 + PE2) correlates with change in mPAP. ** $\mathrm{P}<0.01$; ****, $\mathrm{P}<0.0001$. B, baseline; PE1, post-clot injection 1; PE2, post-clot injection 2. n.s., not significant; MAP, mean arterial pressure; mPAP, mean pulmonary artery pressure; $\mathrm{PaCO}_{2}$, partial pressure of arterial carbon dioxide; $\mathrm{PaO}_{2}$, partial pressure of arterial oxygen; HR, heart rate; SEM, standard error of the mean.

parameters post-clot injection except for HR, which gradually normalized over time (Table S2). MAP (mmHg) remained stable (B: 57.5 $\pm 5.1 v s$. $\mathrm{PE} 1: 59.0 \pm 9.1, \mathrm{P}=0.918$ vs. $\mathrm{PE2}$ : $60.9 \pm 9.6, \mathrm{P}=0.664)$. This data is also shown in Figure 5A. Blood clot volume (mL) (PE1: $5.9 \pm 1.7$ vs. PE1 + PE2: 13.2 \pm 4.4$)$ correlated well with mPAP change $\left[\mathrm{mPAP}_{\mathrm{PE} 1}-\mathrm{mPAP}_{\mathrm{B}}\right.$ : Pearson correlation coefficient $(\mathrm{r})$ $=0.695, \mathrm{P}=0.025 ; \mathrm{mPAP}_{\mathrm{PE}_{2}}-\mathrm{mPAP}_{\mathrm{B}}: \mathrm{r}=0.606, \mathrm{P}=0.063$, Figure 5B].

All animals survived the procedure; no pig was excluded from the study. No complications were observed.

\section{Conclusions}

The findings of the study data support the feasibility of pulmonary emboli generation with EBUS-TBNI. Development of pulmonary hypertension (mPAP $>25 \mathrm{mmHg}$ ) indicates these emboli were physiologically significant. The hemodynamic response was observed immediately after clot injection. These hemodynamic 


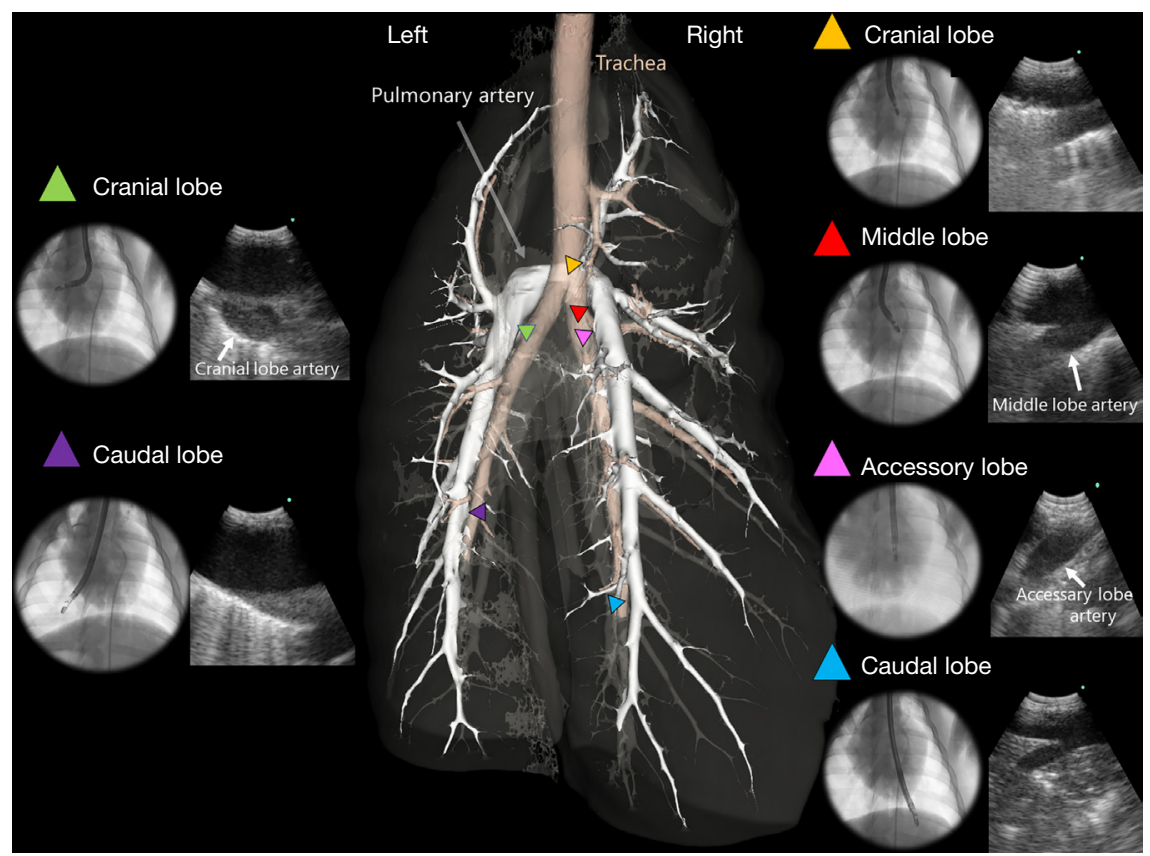

Figure $63 \mathrm{D}$ reconstruction of pig lung anatomy. Virtual model of the bronchial and arterial tree demonstrate pulmonary artery access to the right cranial lobe artery (yellow), middle lobe artery (red), accessary lobe artery (pink), right caudal lobe artery (blue), left cranial lobe artery (green) and left caudal lobe artery (purple) using EBUS. EBUS, endobronchial ultrasound.

parameters didn't show remarkable change except for HR over time (Table S2).

Pig lung anatomy differs slightly from humans. The left lung consists of cranial and caudal lobes that are equivalent to the left upper and lower lobes, respectively. The left cranial lobe bronchus bifurcates into cranial and caudal segmental bronchi, akin to the left upper/ lingular division. The right lung has four lobes: cranial, middle, accessory, and caudal. The right cranial lobe is connected to a tracheal bronchus. The middle bronchus arises from the caudal aspect and the accessory bronchus arises from the medial aspect of the right main bronchus, respectively. The right caudal lobe arises from the right caudal bronchus. Lobar arterial supply parallels the bronchial tree (8). Based on our experience, EBUS reasonably provided access to all lobar bronchi of pigs with lungs and vessels of similar size to humans, albeit not identical (Figure 6). Researchers can further modify model parameters through control of the injected clot volume at each site or employing multiple injection sites. This would allow replication of the full-spectrum of PE, including submassive and massive PE. To our knowledge, all existing animal lung models have significant differences from humans. Therefore, the differences between the lung anatomy in our model and human anatomy is no different from the differences seen in any other animal $\mathrm{PE}$ model—despite those differences, animal research will continue to play a critical role in device/drug protocol/ technique development before introduction in human clinical trials. Often, these animal models may be the only way to provide clinical trial participants with meaningful insight into the potential safety and efficacy profile of the intervention before enrolling.

The study has several limitations. First, clots were formed ex vivo, and may therefore not have the same properties as clot formed in vivo. Second, TBNI may not replicate embolism distribution seen clinically or with models using central venous catheter-based injection. Moreover, clot size and shape formed via an EBUS-TBNI approach are likely less 'clinically realistic' than those formed via central vessel access. Third, BNP, troponin analysis, and echocardiography were not performed in this study. Therefore, stratifying pigs as stable (low-risk) or submassive (intermediate-risk) was not possible; no pigs developed profound hemodynamic instability that would otherwise be consistent with massive (high-risk) PE. These tests would be needed to more fully correlate the animal model to clinical presentations. Fourth, only the lower 
lobes were used; although the EBUS bronchoscope could access all lobar bronchi, limitations with bronchoscope size, angulation range, and white light visualization does make accessing other lobes somewhat more challenging. Finally, our model was not compared with traditional models, whereby clots are delivered by central venous cannulation. Comparing the two approaches from the perspective of clot distribution, hemodynamic and biochemical parameters, and complications, will be needed in future studies.

Our model therefore has greatest utility for early-stage investigation, where more consistent models facilitate device optimization prior to evaluation in more heterogeneous models.

In conclusion, EBUS-TBNI allows precise, reliable, and minimally invasive generation of pulmonary emboli in pigs. This model may serve as an important tool for investigation of new diagnostic and therapeutic approaches to PE.

\section{Acknowledgments}

Funding: This work was supported by a research grant from Olympus Corporation. The sponsor had no role in the study design, conduct of the study, data collection and interpretation, preparation of the report, review of the report, or approval of the report.

\section{Footnote}

Reporting Checklist: The authors have completed the ARRIVE reporting checklist (available at https://jtd. amegroups.com/article/view/10.21037/jtd-21-1242/rc).

Data Sharing Statement: Available at https://jtd.amegroups. com/article/view/10.21037/jtd-21-1242/dss

Peer Review File: Available at https://jtd.amegroups.com/ article/view/10.21037/jtd-21-1242/prf

Conflicts of Interest: All authors have completed the ICMJE uniform disclosure form (available at https://jtd.amegroups. com/article/view/10.21037/jtd-21-1242/coif). KY reports that this work was supported by a research grant from Olympus Corporation, and $\mathrm{KY}$ is a consultant for Olympus. The other authors have no conflicts of interest to declare.
Ethical Statement: The authors are accountable for all aspects of the work in ensuring that questions related to the accuracy or integrity of any part of the work are appropriately investigated and resolved. Experiments were performed under a project license (No. AUP5928) granted by the University Health Network Animal Care Committee, in compliance with the institutional guidelines for the care and use of animals. A protocol was prepared before the study without registration.

Open Access Statement: This is an Open Access article distributed in accordance with the Creative Commons Attribution-NonCommercial-NoDerivs 4.0 International License (CC BY-NC-ND 4.0), which permits the noncommercial replication and distribution of the article with the strict proviso that no changes or edits are made and the original work is properly cited (including links to both the formal publication through the relevant DOI and the license). See: https://creativecommons.org/licenses/by-nc$\mathrm{nd} / 4.0 /$.

\section{References}

1. Barbash IM, Schenke WH, Halabi M, et al. Experimental model of large pulmonary embolism employing controlled release of subacute caval thrombus in swine. J Vasc Interv Radiol 2011;22:1471-7.

2. Schultz J, Andersen A, Gade IL, et al. A porcine invivo model of acute pulmonary embolism. Pulm Circ 2018;8:2045893217738217.

3. Kjaergaard B, Kristensen SR, Risom M, et al. A porcine model of massive, totally occlusive, pulmonary embolism. Thromb Res 2009;124:226-9.

4. Zhao LB, Jia ZY, Lu GD, et al. Establishment of a canine model of acute pulmonary embolism with definite right ventricular dysfunction through introduced autologous blood clots. Thromb Res 2015;135:727-32.

5. Khan F, Anker CJ, Garrison G, et al. Endobronchial ultrasound-guided transbronchial needle injection for local control of recurrent non-small cell lung cancer. Ann Am Thorac Soc 2015;12:101-4.

6. Kinsey CM. Endobronchial ultrasound-guidedtransbronchial needle injection for direct therapy of lung cancer. AME Med J 2018;3:74.

7. Mori V, Roy GS, Bates JHT, et al. Cisplatin 
Pharmacodynamics Following Endobronchial UltrasoundGuided Transbronchial Needle Injection into Lung Tumors. Sci Rep 2019;9:6819.

8. Judge EP, Hughes JM, Egan JJ, et al. Anatomy and

Cite this article as: Inage T, Fujino K, Motooka Y, Ishiwata T, Ujiie H, Gregor A, Bernards N, Chan HHL, Chen Z, Aragaki M, Kinoshita T, Effat A, Yoshino I, Yasufuku K. Development of a minimally invasive pulmonary porcine embolism model via endobronchial ultrasound. J Thorac Dis 2022;14(2):238-246. doi: 10.21037/jtd-21-1242 bronchoscopy of the porcine lung. A model for translational respiratory medicine. Am J Respir Cell Mol Biol 2014;51:334-43. 


\section{Supplementary}

Table S1 Summary of clot size, distribution, and pig weight

\begin{tabular}{|c|c|c|c|c|c|}
\hline \multirow{2}{*}{ Case } & \multirow{2}{*}{ BW $(\mathrm{kg})$} & \multicolumn{2}{|c|}{ PE1 } & \multicolumn{2}{|c|}{ PE2 } \\
\hline & & Volume $(\mathrm{mL})$ & Site & Volume $(\mathrm{mL})$ & Site \\
\hline 1 & 48 & 5 & LLL & 6 & RLL \\
\hline 2 & 35 & 5 & LLL & 7 & RLL \\
\hline 3 & 33 & 8 & RLL & 5 & LLL \\
\hline 4 & 30 & 10 & LLL & 7 & RLL \\
\hline 5 & 32 & 9 & RLL & 10 & RLL \\
\hline 6 & 31 & 3 & RLL & 3 & LLL \\
\hline 7 & 31 & 7 & RLL & 7 & LLL \\
\hline 8 & 32 & 7 & LLL & 7 & RLL \\
\hline 9 & 31 & 4 & RLL & 5 & LLL \\
\hline 10 & 31 & 5 & RLL & 13 & LLL \\
\hline
\end{tabular}

PE1 and PE2, pulmonary embolism. BW, body weight; LLL, left lower lobe pulmonary artery; RLL, right lower lobe pulmonary artery.

Table S2 Summary of hemodynamic measurements

\begin{tabular}{|c|c|c|c|c|c|c|}
\hline Parameters & Baseline & PE1 & PE2 & $30 \mathrm{~min}$ & $60 \mathrm{~min}$ & $120 \mathrm{~min}$ \\
\hline MAP (mmHg) & $57.5 \pm 5.1$ & $59.0 \pm 9.1$ & $60.9 \pm 9.6$ & $59.9 \pm 8.7$ & $56.8 \pm 10.7$ & $57.4 \pm 4.8$ \\
\hline $\mathrm{mPAP}(\mathrm{mmHg})$ & $16.6 \pm 5.6$ & $24.5 \pm 7.6$ & $26.9 \pm 6.7$ & $27.3 \pm 4.9$ & $28.9 \pm 7.2$ & $27.2 \pm 5.6$ \\
\hline $\mathrm{SpO}_{2}(\%)$ & $97.6 \pm 1.4$ & $95.8 \pm 1.8$ & $94.8 \pm 2.5$ & $96.8 \pm 1.2$ & $96.6 \pm 1.5$ & $96.4 \pm 1.5$ \\
\hline
\end{tabular}

PE1 and PE2, 10 min after PE1 and PE2 administration; 30, 60, 120 min, 30, 60, 120 min after PE2 administration. HR, heart rate; MAP, mean arterial pressure; mPAP, mean pulmonary artery pressure; $\mathrm{SpO}_{2}$, oxygen saturation. 